\title{
IRRATIONALITY OF LIMITS OF QUICKLY CONVERGENT ALGEBRAIC NUMBERS SEQUENCES
}

\author{
A. V. NABUTOVSKY
}

(Communicated by Larry J. Goldstein)

\begin{abstract}
We present criteria for irrationality of limits of convergent sequences of rational numbers, algebraic numbers of the same degree and of strictly increasing degrees.

The criterion for irrationality of limits of a sequence of rational numbers has the form of an infinite system of inequalities on successive differences between elements. These inequalities are not strict. If these inequalities are satisfied, then the limit is rational if and only if all inequalities but a finite set of them are satisfied as equalities and the sequence becomes monotonous beginning from some element. So, the criterion permits to see a "border" between rationality and irrationality for some class of quickly convergent sequences.
\end{abstract}

Quickly convergent rational numbers sequences tend to converge to irrational limits. Many special cases of this phenomenon are known (e.g. $[\mathbf{1}, \mathbf{4}])$. In this paper we present sufficient conditions on the convergence of a rational numbers sequence, which are also necessary if some infinite system of inequalities on elements of the sequence is satisfied. The limit of this sequence is irrational, if and only if the infinite set of these inequalities are strict.

Similar criteria for irrationality of limits are obtained for sequences of algebraic numbers of the same degree and for sequences of algebraic numbers with strictly increasing degrees. To apply these criteria, it is sufficient to know only coefficients of minimal polynomials of elements of the sequence. These results are interesting also because it is little known about the approximation of an algebraic number by algebraic numbers of higher degrees (cf. [2] and its exposition in [3]).

Lemma 1 contains the main idea of our proofs. Henceforth, $Z$ denotes the set of integers and $N$ denotes the set of positive integers.

LEMMA 1. Let $\left\{Q_{n}(x)\right\}_{n=1}^{\infty}$ be a sequence of polynomials with integral coefficients and let $a$ be a real number, such that $Q_{n}(a) \neq 0$.

(a) Let all $Q_{n}$ be of the same degree. If there exists an $n_{0}$, such that for $n \geq n_{0}$

$$
\left|Q_{n+1}(a) / Q_{n}(a)\right| \leq 1,
$$

and if the inequality (1) is strict for an infinite number of indices, then the number $a$ is irrational.

(b) Let $\operatorname{deg} Q_{n+1}>\operatorname{deg} Q_{n}$ for sufficiently large values of $n$. If

$$
\lim _{n \rightarrow \infty}\left|Q_{n+1}(a) / Q_{n}(a)\right|^{1 /\left(\operatorname{deg} Q_{k+1}-\operatorname{deg} Q_{n}\right)}=0
$$

then the number $a$ is irrational.

Received by the editors December 20, 1985 and, in revised form, December 22, 1986.

1980 Mathematics Subject Classification (1985 Revision). Primary 11J72; Secondary 40A05. 
Proof. Suppose $a=p / q$ is rational; $p, q \in Z, q>0$. Then $Q_{n}(a)=k_{n} / q^{\operatorname{deg} Q_{n}}$, $k_{n} \in N$. If $Q_{n}$ are of equal degree, then inequality (1) implies $k_{n_{0}+m+1} \leq k_{n_{0}+m}$, and for the infinite set of indices the last inequalities are strict, contrary to our hypothesis, which proves (a). If, for sufficiently large values of $n, \operatorname{deg} Q_{n+1}>$ $\operatorname{deg} Q_{n}$, then

(3) $\left|Q_{n+1}(a) / Q_{n}(a)\right|^{1 /\left(\operatorname{deg} Q_{n+1}-\operatorname{deg} Q_{n}\right)}=(1 / q)\left(k_{n+1} / k_{n}\right)^{1 /\left(\operatorname{deg} Q_{n+1}-\operatorname{deg} Q_{n}\right)}$.

Thus (2) and (3) imply that $\lim _{n \rightarrow \infty} k_{n+1} / k_{n}=0$. Hence, there exists $n_{1}$ such that for each $m \in N, k_{n_{1}+m}<k_{n_{1}+m-1}$, which contradiction proves (b).

Below we choose polynomials $Q_{n}$ such that $Q_{n}\left(a_{n}\right)=0$, where $\left\{a_{n}\right\}_{n=1}^{\infty}$ is a sequence of algebraic numbers convergent to $a$. Lemma 2 aims to exclude the limit $a$ from the irrationality criterion and to express it in terms of $a_{n}$.

LEMMA 2. Let $\left\{a_{n}\right\}_{n=1}^{\infty}$ be a convergent sequence of real numbers, let $\lim _{n \rightarrow \infty} a_{n}$ $=a$, and let $c_{n}(n \in N)$ be real numbers such that

$$
\prod_{n=1}^{\infty} c_{n}=+\infty \text {. }
$$

Let $\varepsilon_{n}=\operatorname{sign}\left(\left(a_{n}-a_{n-1}\right)\left(a_{n+1}-a_{n}\right)\right)$. Suppose, that there exists $n_{0} \in N$ such that for each $n \geq n_{0}, c_{n}>1$,

$$
\begin{aligned}
\left|a_{n+1}-a_{n}\right| /\left|a_{n}-a_{n-1}\right| & \leq\left(c_{n+1}-1\right) /\left(c_{n+1}\left(c_{n}-\varepsilon_{n}\right)\right) \\
a_{n} & \neq a_{n-1} .
\end{aligned}
$$

Then, for all $n \geq n_{0}$,

$$
\left|a-a_{n}\right| c_{n} /\left|a-a_{n-1}\right| \leq 1 .
$$

The inequality (7) for some index $n_{1} \geq n_{0}$ is strict if and only if either the sequence $\left\{a_{n}\right\}_{n=n_{1}-1}^{+\infty}$ is not monotoncus or there exists some index $n_{2} \geq n_{1}$ such that the inequality (5) for the index $n_{2}$ is strict.

ProOF. We may write the inequality (5) in the following form:

$$
\left|a_{n}-a_{n-1}\right| /\left(c_{n}-\varepsilon_{n}\right) \geq c_{n+1}\left|a_{n+1}-a_{n}\right| /\left(c_{n+1}-1\right) .
$$

The inequality (8) implies

$$
\begin{aligned}
& \frac{c_{n+1}\left(a_{n+1}-a_{n}\right)}{c_{n+1}+\operatorname{sign}\left(a_{n+1}-a_{n}\right)} \geq-\frac{\left|a_{n}-a_{n-1}\right|}{c_{n}+\operatorname{sign}\left(a_{n}-a_{n-1}\right)}, \\
& \frac{c_{n+1}\left(a_{n+1}-a_{n}\right)}{c_{n+1}-\operatorname{sign}\left(a_{n+1}-a_{n}\right)} \leq \frac{\left|a_{n}-a_{n-1}\right|}{c_{n}-\operatorname{sign}\left(a_{n}-a_{n-1}\right)} .
\end{aligned}
$$

(The inequalities may be verified by elementary calculations for each combination of signs of $\left(a_{n}-a_{n-1}\right),\left(a_{n+1}-a_{n}\right)$ separately; $a_{n}-a_{n-1} \neq 0, a_{n+1}-a_{n} \neq 0$ by (6).) Adding $\left(a_{n}-a_{n-1}\right)$ to both sides of inequalities (9) and (10) and substituting $\left(a_{n}-a_{n-1}\right) \operatorname{sign}\left(a_{n}-a_{n-1}\right)$ for $\left|a_{n}-a_{n-1}\right|$, we have

$$
\begin{aligned}
& \frac{c_{n+1}\left(a_{n+1}-a_{n}\right)}{c_{n+1}+\operatorname{sign}\left(a_{n+1}-a_{n}\right)}+\left(a_{n}-a_{n-1}\right) \geq \frac{c_{n}\left(a_{n}-a_{n-1}\right)}{c_{n}+\operatorname{sign}\left(a_{n}-a_{n-1}\right)}, \\
& \frac{c_{n+1}\left(a_{n+1}-a_{n}\right)}{c_{n+1}-\operatorname{sign}\left(a_{n+1}-a_{n}\right)}+\left(a_{n}-a_{n-1}\right) \leq \frac{c_{n}\left(a_{n}-a_{n-1}\right)}{c_{n}-\operatorname{sign}\left(a_{n}-a_{n-1}\right)} .
\end{aligned}
$$


We may write (11) as

$$
d_{n+1} \geq d_{n}+\left(a_{n-1}-a_{n}\right), \quad e_{n+1} \leq e_{n}+\left(a_{n-1}-a_{n}\right),
$$

where

$$
\begin{aligned}
& d_{n}=c_{n}\left(a_{n}-a_{n-1}\right) /\left(c_{n}+\operatorname{sign}\left(a_{n}-a_{n-1}\right)\right), \\
& e_{n}=c_{n}\left(a_{n}-a_{n-1}\right) /\left(c_{n}-\operatorname{sign}\left(a_{n}-a_{n-1}\right)\right) .
\end{aligned}
$$

Applying each of these inequalities twice for indices $n$ and $n+1$, one obtains

$$
\begin{aligned}
& d_{n+2} \geq d_{n}+\left(a_{n-1}-a_{n}\right)+\left(a_{n}-a_{n+1}\right), \\
& e_{n+2} \leq e_{n}+\left(a_{n-1}-a_{n}\right)+\left(a_{n}-a_{n+1}\right) .
\end{aligned}
$$

Proceeding inductively one obtains

$$
d_{n+k} \geq d_{n}+a_{n-1}-a_{n+k-1}, \quad e_{n+k} \leq e_{n}+a_{n-1}-a_{n+k-1}
$$

for all $k \in N$. We now prove that

$$
\lim _{k \rightarrow+\infty} d_{k}=\lim _{k \rightarrow+\infty} e_{k}=0 .
$$

Indeed, $g_{n} \equiv \max \left(\left|d_{n}\right|,\left|e_{n}\right|\right)=c_{n}\left|a_{n}-a_{n-1}\right| /\left(c_{n}-1\right)$. It follows from (8) that for all $n>n_{0} g_{n+1} \leq g_{n} / c_{n}$. So for each $n \geq n_{0}+1$

$$
g_{n} \leq g_{n_{0}} / \prod_{i=n_{0}}^{n-1} c_{i}
$$

This inequality together with (4) yields $\lim _{k \rightarrow+\infty} g_{k}=0$, which implies (13). Passing to the limit in (12) as $k \rightarrow+\infty$ we have

$$
d_{n}+a_{n-1}-a \leq 0, \quad e_{n}+a_{n-1}-a \geq 0 .
$$

Now note that $\operatorname{sign}\left(a_{n}-a_{n-1}\right)=\operatorname{sign}\left(a-a_{n-1}\right)$ for each $n \geq n_{0}$ by (14), because $\operatorname{sign} d_{n}=\operatorname{sign} e_{n}=\operatorname{sign}\left(a_{n}-a_{n-1}\right)$. Using this fact and substituting expressions for $d_{n}, e_{n}$ into (14), after elementary calculations one obtains

$$
\left(a-a_{n}\right) c_{n} \geq-\left|a-a_{n-1}\right|, \quad\left(a-a_{n}\right) c_{n} \leq\left|a-a_{n-1}\right| .
$$

Obviously (15) implies (7). It follows easily from the proof that (7) is an equality if and only if, for all indices $m \geq n,(4)$ is equality and the sequence $\left\{a_{k}\right\}_{k=n-1}^{\infty}$ is monotonous (because otherwise we use a strict inequality (9) or (10) for some index in the process of demonstration). This completes the proof of Lemma 2.

THEOREM 1. Let $a_{n}=p_{n} / q_{n}, p_{n}, q_{n} \in Z, q_{n+1}>q_{n}>0, \lim _{n \rightarrow+\infty} a_{n}=a$. Let $\varepsilon_{n}=\operatorname{sign}\left(\left(a_{n+1}-a_{n}\right)\left(a_{n}-a_{n-1}\right)\right)$. Suppose there exists $n_{0} \in N$ such that, for all $n \geq n_{0}, a_{n} \neq a_{n-1}$ and

$$
\left|a_{n+1}-a_{n}\right| /\left|a_{n}-a_{n-1}\right| \leq\left(q_{n+1}-q_{n}\right) q_{n-1} /\left(q_{n+1}\left(q_{n}-\varepsilon_{n} q_{n-1}\right)\right) .
$$

Let $N_{1} \subset N$ be the set of indices $n$ such that the inequality (16) is strict, and let $N_{2} \subset N$ be the set of indices $n$ such that $\varepsilon_{n}=-1 ; N^{\prime}=N_{1} \cup N_{2}$. Then $a$ is irrational if and only if $N^{\prime}$ is infinite.

PrOOF. Let $c_{n}=q_{n} / q_{n-1}$. Obviously, $\prod_{n=n_{0}}^{\infty} c_{n}=+\infty$. By Lemma 2,

$$
\left|a_{n+1}-a_{n}\right| q_{n} /\left|a_{n}-a_{n-1}\right| q_{n-1} \leq 1 \text {. }
$$


If $N^{\prime}$ is finite then, by Lemma 2 , for some index $n^{\prime},(17)$ becomes an equality. This implies immediately rationality of $a$. If $N^{\prime}$ is infinite then, by Lemma 2 , for $n>n_{0}$ all inequalities (17) are strict. But the system of strict inequalities (17) coincides with the system of inequalities (1) for polynomials $Q_{n}(x)=q_{n} x-p_{n}$. Hence, by Lemma 1, $a$ is irrational. Q.E.D.

If one applies Theorem 1 to a sequence of partial sums of a series (or partial products of an infinite product) one may suppose that the denominators of those partial sums (or products) are equal to products of denominators of the terms. It yields (after simple calculations) Corollaries 1 and 2 .

COROLlaRY 1. Let $a=\sum_{n=1}^{\infty} x_{n} / y_{n}, x_{n}, y_{n} \in Z, y_{n} \geq 2$. Let there exist $n_{0} \in N$ such that, for each $n \geq n_{0}$,

$$
\frac{\left|x_{n+1}\right|}{y_{n+1}-1} \leq \frac{\left|x_{n}\right|}{y_{n}\left(y_{n}-\operatorname{sign}\left(x_{n} x_{n+1}\right)\right)}
$$

Let $N_{1}$ be the set of indices $n$ such that inequality (18) is strict and let $N_{2}$ be the set of indices $n$ such that $\operatorname{sign}\left(x_{n+1}\right) \neq \operatorname{sign}\left(x_{n}\right)$. Then $a$ is irrational if and only if $N_{1} \cup N_{2}$ is infinite.

EXAmple 1 (J. J. Sylvester, [4]). Let $n_{1} \in N \backslash\{1\}, n_{k+1}=n_{k}^{2}-n_{k}+1$. Then $\sum_{k=1}^{\infty} 1 / n_{k}$ is rational by Corollary 1 (and is equal to $1 /\left(n_{1}-1\right)$ ). Let $m_{1} \in N \backslash\{1\}, m_{k+1}=m_{k}^{2}-m_{k}+2$. Then $\sum_{k=1}^{\infty} 1 / m_{k}$ is irrational by Corollary 1.

COROLlaRY 2. Let $a=\prod_{n=1}^{\infty}\left(1+x_{n} / y_{n}\right), x_{n}, y_{n} \in Z, y_{n} \geq 2, x_{n}+y_{n}>0$. Suppose there exists $n_{0} \in N$ such that, for each $n \geq n_{0}$,

$$
\frac{\left|x_{n+1}\right|}{y_{n+1}-1} \leq \frac{\left|x_{n}\right|}{\left(x_{n}+y_{n}\right)\left(y_{n}-\operatorname{sign}\left(x_{n+1}\right)\right)} \text {. }
$$

Let $N_{1}$ be the set of indices $n$ such that inequality (19) is strict, and let $N_{2}$ be the set of indices $n$ such that $\operatorname{sign}\left(x_{n+1}\right) \neq \operatorname{sign}\left(x_{n}\right)$. Then $a$ is irrational if and only if $N_{1} \cup N_{2}$ is infinite.

Example 2 (G. CANTOR, CF . [4]). For each $m \in N \backslash\{1\}$,

$$
\prod_{n=1}^{\infty}\left(1+1 /\left(m^{2^{n}}-r\right)\right)
$$

is irrational for each positive integer $r \neq m^{2^{n}}$ by Corollary $2 . \Pi_{n=1}^{\infty}\left(1+1 / m^{2^{n}}\right)$ is rational by Corollary 2 (and is equal to $m /(m-1)$ ).

COROLLARY 3. Let $P_{n}(x)$ be polynomials with integral coefficients, $t_{n}=x_{n} / y_{n}$, $x_{n} \in Z \backslash\{0\}, y_{n} \in N \backslash\{1\}, \operatorname{deg} P_{n}=d_{n}, \lim _{n \rightarrow \infty} y_{n}=+\infty$. Let $x_{0}$ be rational and $P_{n}\left(x_{0}\right) \neq 0$ for all $n$. Let $a=\sum_{n=1}^{\infty} t_{n} P_{n}\left(x_{0}\right)$.

(a) Let all $P_{n}$ be of the same degree. If

$$
\limsup _{n \rightarrow \infty}\left|t_{n+1} y_{n} P_{n+1}\left(x_{0}\right) / t_{n} P_{n}\left(x_{0}\right)\right|<1,
$$

then $a$ is irrational. 
(b) Let, for sufficiently large values of $n, d_{n+1}>d_{n}$. If

$$
\lim _{n \rightarrow \infty}\left|t_{n+1} y_{n} P_{n+1}\left(x_{0}\right) /\left(t_{n} P_{n}\left(x_{0}\right)\right)\right|^{1 /\left(d_{n+1}-d_{n}\right)}=0
$$

then $a$ is irrational.

Proof. Let $x_{0}=p / q$. Define

$$
a_{n}=\sum_{k=1}^{n} t_{k} P_{k}\left(x_{0}\right), \quad l_{n}=\max d_{k}, \quad q_{n}=\left(\prod_{k=1}^{n} y_{k}\right) q^{l_{n}} .
$$

Strict inequalities (16) then follow from the inequality

$$
\left|a_{n+1}-a_{n}\right| /\left|a_{n}-a_{n-1}\right|<q_{n-1}\left(q_{n+1}-q_{n}\right) /\left(\left(q_{n}+q_{n-1}\right) q_{n+1}\right) .
$$

But

$$
\frac{q_{n-1}\left(q_{n+1}-q_{n}\right)}{\left(q_{n}+q_{n-1}\right) q_{n+1}}=\frac{\left(q_{n-1} / q_{n}\right)\left(1-q_{n} / q_{n+1}\right)}{1+q_{n-1} / q_{n}}
$$

and, by definition of $q_{n}$,

$$
\lim _{n \rightarrow \infty} q_{n-1} / q_{n}=0 .
$$

So inequalities (16) follow from the inequality

$$
\limsup _{n \rightarrow \infty}\left|a_{n+1}-a_{n}\right| q_{n} /\left(\left|a_{n}-a_{n-1}\right| q_{n-1}\right)<1 .
$$

It can be easily verified that (20) and (21) imply (22). So by Theorem 1, Corollary 3 is proved.

EXAMPLE 3. Let $F(x)=\sum_{n=0}^{\infty} b_{n} x 2^{n}$, where

$$
b_{0}=\sum_{k=1}^{\infty} 2^{-2^{k} k^{2}}, \quad b_{n}=(-1)^{n} \sum_{k=n}^{\infty} 2^{-2^{k} k^{2}}
$$

for $n \geq 1$. Then $F(x)$ assumes irrational values for all rational $x$. For $x \neq 1$ this follows from Corollary 3(b), for $x=1$ from Corollary 1 .

Below we obtain results for sequences of algebraic numbers similar to the part of Theorem 1, which states irrationality of the limits.

LEMMA 3. Let $\left\{a_{n}\right\}_{n=1}^{\infty}$ be a convergent sequence of real numbers, $\lim _{n \rightarrow \infty} a_{n}=$ a. Let $\left\{c_{n}\right\}_{n=1}^{\infty}$ be a sequence of real numbers such that

$$
\lim _{n \rightarrow \infty} c_{n}=+\infty
$$

and let $0<u_{n} \leq 1$ for all $n \in N$. If the limit in the left side of (24) is finite, then (24) $\limsup _{n \rightarrow \infty} c_{n}\left(\left|a_{n+1}-a_{n}\right| /\left|a_{n}-a_{n-1}\right|\right)^{u_{n}}=\limsup _{n \rightarrow \infty} c_{n}\left(\left|a-a_{n}\right| /\left|a-a_{n-1}\right|\right)^{u_{n}}$.

ProOF. From the finiteness of the limit in the left part of (24) and the condition (23), it follows that

$$
\lim _{n \rightarrow \infty}\left|a_{n+1}-a_{n}\right| /\left|a_{n}-a_{n-1}\right|=0 .
$$

But

$$
\frac{\left|a-a_{n-1}\right|}{\left|a-a_{n}\right|}=\left|1+\frac{a_{n}-a_{n-1}}{\sum_{k=1}^{\infty}\left(a_{n+k}-a_{n+k-1}\right)}\right| .
$$


Thus, if for all sufficiently large values of $n$,

$$
\left|a_{n+1}-a_{n}\right| /\left|a_{n}-a_{n-1}\right|<\varepsilon
$$

then, for those values of $n$,

$$
\frac{\left|a-a_{n-1}\right|}{\left|a-a_{n}\right|} \geq \frac{\left|a_{n}-a_{n-1}\right|}{\left|a_{n}-a_{n-1}\right|(\varepsilon /(1-\varepsilon))}-1=\frac{1}{\varepsilon}-2 .
$$

Hence,

$$
\lim _{n \rightarrow \infty}\left|a-a_{n}\right| /\left|a-a_{n-1}\right|=0
$$

and

$$
\begin{aligned}
& \limsup _{n \rightarrow \infty} c_{n}\left(\left|a_{n+1}-a_{n}\right| /\left|a_{n}-a_{n-1}\right|\right)^{u_{n}} \\
& \quad=\limsup _{n \rightarrow \infty} c_{n}\left(\left|\left(\left(a-a_{n}\right)-\left(a-a_{n+1}\right)\right) /\left(\left(a-a_{n-1}\right)-\left(a-a_{n}\right)\right)\right|\right)^{u_{n}} \\
& \quad=\limsup _{n \rightarrow \infty} c_{n}\left(\left|a-a_{n}\right|(1+0(1)) /\left(\left|a-a_{n-1}\right|(1+0(1))\right)\right)^{u_{n}} \\
& \quad=\limsup _{n \rightarrow \infty} c_{n}\left(\left|a-a_{n}\right| /\left|a-a_{n-1}\right|\right)^{u_{n}} \text {. Q.E.D. }
\end{aligned}
$$

REMARK. If $\lim _{n \rightarrow \infty} c_{n}\left(\left|a_{n+1}-a_{n}\right| /\left|a_{n}-a_{n-1}\right|\right)^{u_{n}}=0$, then the condition (23) can be replaced by

$$
\liminf _{n \rightarrow \infty} c_{n}>0
$$

This condition still yields (25), and the proof of the resulting modified lemma coincides with the proof of Lemma 3 just given.

THEOREM 2. Let $\left\{a_{n}\right\}_{n=1}^{\infty}$ be a convergent sequence of algebraic numbers

$$
\lim _{n \rightarrow \infty} a_{n}=a, \quad a_{n} \neq a .
$$

Let $\left\{Q_{n}\right\}_{n=1}$ be a sequence of polynomials with integral coefficients such that $Q_{n}\left(a_{n}\right)$ $=0$. Let $\operatorname{deg} Q_{n}=d_{n}$. Let

$$
\lim _{n \rightarrow \infty} \min _{x \in\left[s_{n}, t_{n}\right]} Q_{n}^{\prime}(x) / \max _{x \in\left[s_{n}, t_{n}\right]} Q_{n}^{\prime}(x)=1,
$$

where $\left[s_{n}, t_{n}\right]$ denotes $\left[a_{n}, a\right]$ for $a_{n}<a$, or $\left[a, a_{n}\right]$ for $a_{n}>a$.

(a) If all $Q_{n}$ 's are of the same degree,

$$
\lim _{n \rightarrow \infty}\left|Q_{n+1}^{\prime}\left(a_{n+1}\right)\right| /\left|Q_{n}^{\prime}\left(a_{n}\right)\right|=+\infty
$$

and

$$
\limsup _{n \rightarrow \infty}\left(\left|Q_{n}^{\prime}\left(a_{n}\right)\right| /\left|Q_{n-1}^{\prime}\left(a_{n-1}\right)\right|\right)\left|a_{n+1}-a_{n}\right| /\left|a_{n}-a_{n-1}\right|<1,
$$

then $a$ is irrational.

(b) If, for sufficiently large values of $n, d_{n+1}>d_{n}$,

$$
\liminf _{n \rightarrow \infty}\left(\left|Q_{n+1}^{\prime}\left(a_{n+1}\right)\right| /\left|Q_{n}^{\prime}\left(a_{n}\right)\right|\right)^{1 /\left(d_{n+1}-d_{n}\right)}>0
$$

and

$$
\lim _{n \rightarrow \infty}\left(\left(\left|Q_{n}^{\prime}\left(a_{n}\right)\right| /\left|Q_{n-1}^{\prime}\left(a_{n-1}\right)\right|\right)\left|a_{n+1}-a_{n}\right| /\left|a_{n}-a_{n-1}\right|\right)^{1 /\left(d_{n}-d_{n-1}\right)}=0,
$$

then $a$ is irrational. 
ProOF. Note at first that

$$
\begin{aligned}
\left|Q_{n+1}(a)\right| /\left|Q_{n}(a)\right| & =\left|Q_{n+1}(a)-Q_{n+1}\left(a_{n+1}\right)\right| /\left|Q_{n}(a)-Q_{n}\left(a_{n}\right)\right| \\
& =\left|Q_{n+1}^{\prime}\left(\xi_{n+1}\right)\right|\left|a-a_{n+1}\right| /\left(\left|Q_{n}^{\prime}\left(\xi_{n}\right)\right|\left|a-a_{n}\right|\right),
\end{aligned}
$$

where $\xi_{n} \in\left(s_{n}, t_{n}\right)$. Now

$$
\begin{aligned}
\left|Q_{n+1}^{\prime}\left(\xi_{n+1}\right)\right| /\left|Q_{n}^{\prime}\left(\xi_{n}\right)\right|= & \left(\left|Q_{n+1}^{\prime}\left(\xi_{n+1}\right)\right| /\left|Q_{n+1}^{\prime}\left(a_{n+1}\right)\right|\right) \\
& \times\left(\left|Q_{n+1}^{\prime}\left(a_{n+1}\right)\right| /\left|Q_{n}^{\prime}\left(a_{n}\right)\right|\right)\left(\left|Q_{n}^{\prime}\left(a_{n}\right)\right| /\left|Q_{n}^{\prime}\left(\xi_{n}\right)\right|\right) .
\end{aligned}
$$

From (31) and (32) it can be seen, by condition (27), that the irrationality criterion of Lemma 1 in case (a) follows from

$$
\limsup _{n \rightarrow \infty}\left(\left|Q_{n+1}^{\prime}\left(a_{n+1}\right) /\right| Q_{n}^{\prime}\left(a_{n}\right) \mid\right)\left|a-a_{n+1}\right| /\left|a-a_{n}\right|<1
$$

and in case (b) it is equivalent to

$$
\lim _{n \rightarrow \infty}\left(\left|Q_{n+1}^{\prime}\left(a_{n+1}\right)\right|\left|a-a_{n+1}\right| /\left(\left|Q_{n}^{\prime}\left(a_{n}\right)\right|\left|a-a_{n}\right|\right)\right)^{1 /\left(d_{n+1}-d_{n}\right)}=0 .
$$

But (28), (28'), (29), and (30) imply (33) and (34) by Lemma 3 and the remark after it. So Theorem 2 is proved.

ACKNOWLEDGMENTS. I would like to thank Professor A. I. Fet and Professor N. I. Feldman for encouragement.

ADDED IN PROOF. Recently the author was informed that Corollary 2 and several particular cases of Corollary 1 were proven by A. Oppenheim by a different method (for references see the book of J. Galambos, Representations of real numbers by infinite series, Springer-Verlag, Berlin, 1976).

\section{REFERENCES}

1. P. Erdos, Problem 4321, Amer. Math. Monthly 64 (1957), 47.

2. M. Mignotte, Approximation des nombres algébriques par des nombres algébriques de grand degré, Ann. Fac. Sci. Toulouse 1 (1979), 165-170.

3. W. Schmidt, Diophantine approximation, Springer-Verlag, Berlin, 1980.

4. D. Perron, Irrationalzahlen, DeGruyter, Berlin, 1960.

ZolotodolinskAYA, 17, APT. 23, NOVOSIBIRSK, 630072, USSR

Current address: Department of Theoretical Mathematics, The Weizmann Institute of Science, Rehovot, 76100, Israel 